\title{
Avaliação dos algoritmos de Picard-Krylov na solução da equação de Richards
}

\author{
Marcelo Xavier Guterres \\ Engenharia de Produção, Laboratório de modelagem e Simulação, LMSC, UNIPAMPA, \\ 96413-170, Bagé, RS \\ E-mail: m.guterres@unipampa.edu.br. \\ João Flávio Vieira Vasconcellos, Antônio José da Silva Neto, \\ UERJ- IPRJ - Engenharia Mecânica \\ 28630-050, Nova Friburgo, RJ \\ E-mail: jflavio@iprj.uerj.br, ajsneto@iprj.uerj.br.
}

\begin{abstract}
Resumo: No artigo presente aproximou-se uma solução para a equação diferencial parcial de Richards pelo método dos volumes finitos em duas dimensões, empregando o método de Picard com maior eficiência computacional. Para tanto, foram utilizadas técnicas iterativas de resolução de sistemas lineares baseados no espaço de Krylov com matrizes pré-condicionadoras com a biblioteca numérica Portable, Extensible Toolkit for Scientific Computation (PETSc). Os resultados indicam que quando se resolve a equação de Richards considerando-se o método de PICARD-KRYLOV, não importando o modelo de avaliação do solo, a melhor combinação para resolução dos sistemas lineares é o método dos gradientes biconjugados estabilizado mais o précondicionador SOR.
\end{abstract}

Palavras-chave: Equação de Richards, Picard-Krylov, PETSc.

\section{Introdução}

A engenharia geotécnica é uma das grandes áreas da engenharia civil que estuda a interação entre as construções realizadas pelo homem ou de fenômenos naturais com o ambiente geológico, que na grande maioria das vezes trata-se de solos parcialmente saturados. Como resultado, o desempenho de obras como estabilização, contenção de barragens, muros de contenção, fundações e estradas estão condicionados a uma correta predição do fluxo de água no interior dos solos, que pode ser alcançada por meio da resolução da equação diferencial parcial de Richards, Eq. 1.

$$
\frac{\partial \theta}{\partial t}=\frac{\partial}{\partial x}\left(K_{x}(\psi) \frac{\partial \psi}{\partial x}\right)+\frac{\partial}{\partial y}\left(K_{y}(\psi) \frac{\partial \psi}{\partial y}\right)+\frac{\partial}{\partial z}\left(K_{z}(\psi) \frac{\partial \psi}{\partial z}\right)-\frac{\partial K_{z}(\psi)}{\partial z}
$$

onde $t$ é o tempo $[t] ; \theta$ é o conteúdo de umidade volumétrico $\left[L^{3} / L^{3}\right] ; \psi$ é a carga devida à pressão $[L] ; K_{x}, K_{y}$ e $K_{z}$ são as condutividades hidráulicas não saturadas $[L / t]$ nas direções espaciais $x, y$ e $z[L]$, respectivamente.

Porém, como a área das regiões a serem estudas com relação à predição do fluxo de água são comumente da ordem de quilômetros quadrados, as soluções dos modelos matemáticos exigem malhas computacionais de grandes proporções, ocasionando sérias limitações associadas aos requisitos de memória computacional e tempo de processamento. A fim de contornar estas limitações, métodos numéricos eficientes devem ser empregados na solução do problema em análise. Portanto, métodos iterativos para solução de sistemas não lineares e lineares esparsos 
de grande porte devem ser utilizados neste tipo de aplicação. Em suma, visto a relevância do tema, esta pesquisa aproximou uma solução para a equação diferencial parcial de Richards pelo método dos volumes finitos em duas dimensões, empregando o método de Picard com maior eficiência computacional. Para tanto, foram utilizadas técnicas iterativas de resolução de sistemas lineares baseados no espaço de Krylov com matrizes pré-condicionadoras com a biblioteca numérica Portable, Extensible Toolkit for Scientific Computation (PETSc) [12].

\section{Modelagem Numérica e Computacional}

Pretende-se nesta seção apresentar os conceitos matemáticos e computacionais envolvidos na elaboração do modelo de aproximação da solução da Equação de Richards. Para tanto, considerar-se-á a equação de Richards baseada em $\psi$, expressa pela Eq. 2.

$$
C(\psi) \frac{\partial \psi}{\partial t}=\frac{\partial}{\partial x}\left(K(\psi) \frac{\partial \psi}{\partial x}\right)+\frac{\partial}{\partial z}\left(K(\psi) \frac{\partial \psi}{\partial z}\right)-\frac{\partial K(\psi)}{\partial z}
$$

em que $C(\psi)=\partial \theta / \partial \psi[1 / L]$ é a capacidade hidráulica específica.

Logo, para solucionar-se numericamente a equação de Richards é imprescindível conhecer as relações entre o conteúdo de umidade volumétrico $(\theta)$, a carga devida à pressão $(\psi)$ e a condutividade hidráulica de um solo não saturado $(K)$, ou seja, $f: \psi \rightarrow \theta, f: \psi \rightarrow K$ e $f: \theta \rightarrow K$. Neste contexto, o modelo mais difundido atualmente é de van Genuchten [9], tanto para $K=f(\psi)$ e $\theta=f(\psi)$. Cujas relações são dadas pelas Eqs. 3 e 4 :

$$
\begin{gathered}
\theta(\psi)=\theta_{r}+\frac{\theta_{s}-\theta_{r}}{\left[1+(\alpha|\psi|)^{n}\right]^{m}} \\
K(\psi)=K s \frac{\left\{1-\left(\alpha|\psi|^{n-1}\right)\left[1+\left(\alpha|\psi|^{n}\right)\right]^{-m}\right\}^{2}}{\left[1+(\alpha|\psi|)^{n}\right]^{m / 2}}
\end{gathered}
$$

onde $\theta_{s}$ é o teor de umidade volumétrico na saturação $\left[L^{3} / L^{3}\right] ; \theta_{r}$ é o teor de umidade volumétrico residual $\left[L^{3} / L^{3}\right]$; $\alpha$ é a função densidade dos tamanhos poros $\left[L^{-1}\right]$; e $m, n$ são parâmetros adimensionais do solo, com $m=1-1 / n$.

Muito embora, apesar das Eqs. 3 e 4 serem mais complexas quando comparadas com outros modelos, elas tem sido vastamente utilizadas em simulações numéricas por ajustar melhor os dados experimentais, acrescido do fato de serem contínuas e diferenciáveis, além disso por incorporarem a teoria de Mualen [14] (modelo estatístico de distribuição de tamanho de poros). Completando, a capacidade hidráulica específica do solo $C(\psi)$ pode ser avaliada analiticamente pela Eq. 5:

$$
C(\psi)=\frac{\partial \theta}{\partial \psi}=\left(-m n \alpha^{n}\right)\left[\frac{\theta_{s}-\theta_{r}}{\left(1+\alpha^{n}|\psi|^{n}\right)^{m+1}}\right]|\psi|^{n} \psi^{-1}
$$

De outro modo, ao se resolver problemas de fluxo em meios porosos uma característica fundamental procurada é a observância da conservação da massa, requisito essencial para que a solução possua coerência física. Então, visto que a conservação das grandezas físicas no nível discreto é uma característica intrínseca do método de volumes finitos (MVF), este método foi escolhido para resolver a equação de Richards, Eq. 2. 


\subsection{Método dos Volumes Finitos}

Neste trabalho a obtenção da equação de discretização do MVF para o problema de fluxo em meios porosos bidimensional transiente foi realizada integrando a Eq. 2, na sua forma divergente sobre um volume elementar, definido pela malha computacional, Fig. 1, no espaço e no tempo.

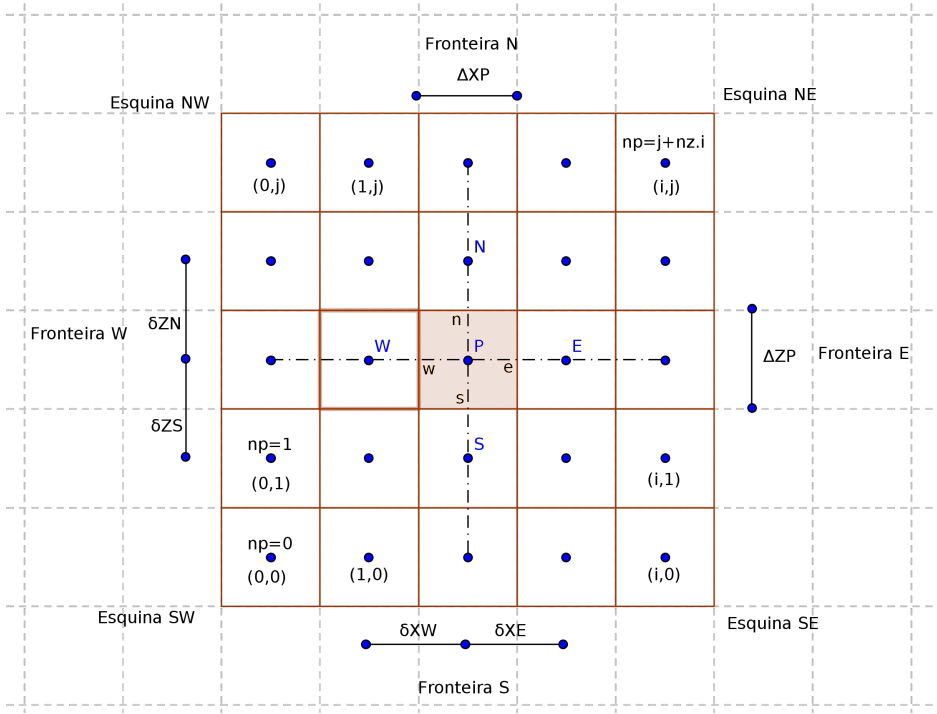

Figura 1: Malha computacional

onde $P, S, N, W$ e $E$ são os nós da malha computacional (centroide do volume de controle); $w$, $e, s$ e $n$ são as interfaces do volume de controle $P$; $n x$ e $n z$ são os números totais de volumes de controle na direção $x$ e $z$, respectivamente; $\Delta Z_{p}$ e $\Delta X_{p}$ são as distâncias entre as interfaces (tamanho do volume de controle); e $n p=i \times n z+j$ é o índice do volume de controle que está sendo integrado, para $i=0,1, \ldots, n x-1$ e $j=0,1, \ldots, n z-1$.

Posto isto, integra-se a equação governante, Eq. 2, sobre cada um dos volumes de controle no domínio do tempo e do espaço para os volumes internos da malha computacional, Fig. 1, ou seja, para $1 \leq i<n x-1$ e $1 \leq j<n z-1$, conforme a Eq. 6 :

$$
\begin{array}{r}
\int_{t}^{t+1} \int_{s}^{n} \int_{w}^{e} C(\psi) \frac{\partial \psi}{\partial t} d x d z d t=\int_{t}^{t+1} \int_{s}^{n} \int_{w}^{e} \frac{\partial}{\partial x}\left(K(\psi) \frac{\partial \psi}{\partial x}\right) d x d z d t+ \\
\int_{t}^{t+1} \int_{s}^{n} \int_{w}^{e} \frac{\partial}{\partial z}\left(K(\psi) \frac{\partial \psi}{\partial z}\right) d x d z d t- \\
\int_{t}^{t+1} \int_{s}^{n} \int_{w}^{e} \frac{\partial K(\psi)}{\partial z} d x d z d t
\end{array}
$$

Cujo resultado da integração da Eq. 6 é dado pela Eq. 7:

$$
\begin{array}{r}
\left.\frac{K_{s}(\psi)}{\Delta Z_{P}} \frac{\partial \psi}{\partial z}\right|_{s}+\left.\frac{K_{w}(\psi)}{\Delta X_{P}} \frac{\partial \psi}{\partial x}\right|_{w}-\left.\frac{K_{e}(\psi)}{\Delta X_{P}} \frac{\partial \psi}{\partial x}\right|_{e}-\left.\frac{K_{n}(\psi)}{\Delta Z_{P}} \frac{\partial \psi}{\partial z}\right|_{n}+\frac{C_{P}\left(\psi_{P}\right) \psi_{P}^{t+1}}{\Delta t}= \\
\frac{C_{P}\left(\psi_{P}\right) \psi_{P}^{t}}{\Delta t}-\frac{K_{n}(\psi)}{\Delta Z_{P}}+\frac{K_{s}(\psi)}{\Delta Z_{P}}
\end{array}
$$

onde $\triangle t$ é o passo tempo, com $t+\Delta t \equiv t+1$. 
Duas questões importantes que se põem no presente momento são: o nível de tempo que será avaliada a variável $\psi$; e como calcular as derivadas nas faces $(w, e, s, n)$ do volume de controle $P$. Para a primeira questão adota-se um esquema de interpolação temporal totalmente implícito, que é incondicionalmente estável do passo temporal. Isto quer dizer que o tamanho do passo de tempo não interfere na resolução da equação diferencial, assim a variável $\psi$ será avaliada em $t+1$. Com relação ao segundo ponto, ao calcular-se as derivadas nas faces do volume de controle $(w, e, s, n)$ na Eq. 7, considera-se um esquema de diferenças centrais, pois é razoável escolher uma função linear entre os pontos nodais obtida através da expansão da série de Taylor, desprezando-se os termos de segunda ordem ou superior.

Então, levando-se estas considerações na Eq. 7 chega-se a Eq. 8:

$$
\begin{gathered}
-\left(\frac{K_{s}^{t+1}\left(\psi_{s}^{t+1}\right)}{\Delta Z_{P} \delta Z_{S}}\right) \psi_{S}^{t+1}-\left(\frac{K_{w}^{t+1}\left(\psi_{w}^{t+1}\right)}{\Delta X_{P} \delta X_{W}}\right) \psi_{W}^{t+1}+\left(\frac{K_{s}^{t+1}\left(\psi_{s}^{t+1}\right)}{\Delta Z_{P} \delta Z_{S}}+\frac{K_{w}^{t+1}\left(\psi_{w}^{t+1}\right)}{\Delta X_{P} \delta X_{W}}+\right. \\
\left.\frac{K_{e}^{t+1}\left(\psi_{e}^{t+1}\right)}{\Delta X_{P} \delta X_{E}}+\frac{K_{n}^{t+1}\left(\psi_{n}^{t+1}\right)}{\Delta Z_{P} \delta Z_{N}}+\frac{C_{p}^{t+1}\left(\psi_{P}^{t+1}\right)}{\Delta t}\right) \psi_{P}^{t+1}-\left(\frac{K_{e}^{t+1}\left(\psi_{e}^{t+1}\right)}{\Delta X_{P} \delta X_{E}}\right) \psi_{E}^{t+1}- \\
-\left(\frac{K_{n}^{t+1}\left(\psi_{n}^{t+1}\right)}{\Delta Z_{P} \delta Z_{N}}\right) \psi_{N}^{t+1}=\frac{C_{P}^{t+1}\left(\psi_{P}^{t+1}\right) \psi_{P}^{t}}{\Delta t}-\frac{K_{n}^{t+1}\left(\psi_{n}^{t+1}\right)}{\Delta Z_{P}}+\frac{K_{s}^{t+1}\left(\psi_{s}^{t+1}\right)}{\Delta Z_{P}}
\end{gathered}
$$

A Eq 8 pode ser definida como a equação algébrica discretizada do fenômeno de fluxo bidimensional de água num meio poroso, válida para os volumes internos da malha computacional, Fig. 1. Podendo ser escrita de maneira mais simplificada conforme a expressão indicada pela Eq. 9:

$$
-A_{S} \psi_{S}^{t+1}-A_{W} \psi_{W}^{t+1}+A_{P} \psi_{P}^{t+1}-A_{E} \psi_{E}^{t+1}-A_{N} \psi_{N}^{t+1}=S_{p}
$$

Porém, é importante que se diga que o resultado da integração da equação de Richards, dado pela Eq. 9 também pode ser válido para os volumes localizados nas fronteiras do domínio, desde que se respeite, as condições de contorno fixadas pelo modelo matemático.

Uma questão importante diz respeito ao cálculo da condutividade hidráulica nas interfaces $K_{s}\left(\psi_{s}\right), K_{n}\left(\psi_{n}\right), K_{w}\left(\psi_{w}\right)$ e $K_{e}\left(\psi_{e}\right)$. De maneira geral, elas podem ser calculadas pela média ponderada, média harmônica ou média geométrica. Neste trabalho optou-se pela média geométrica tal como proposto por Haverkamp e Vauclin [10] e Vasconcellos e Amorim [2].

$$
\begin{array}{cc}
K_{n}\left(\psi_{n}\right)=\sqrt{K_{N}\left(\psi_{N}\right) K_{P}\left(\psi_{P}\right)} & K_{s}\left(\psi_{s}\right)=\sqrt{K_{P}\left(\psi_{P}\right) K_{S}\left(\psi_{S}\right)} \\
K_{w}\left(\psi_{w}\right)=\sqrt{K_{P}\left(\psi_{P}\right) K_{W}\left(\psi_{W}\right)} & K_{e}\left(\psi_{e}\right)=\sqrt{K_{E}\left(\psi_{E}\right) K_{P}\left(\psi_{P}\right)}
\end{array}
$$

Para concluir o problema de predição do fluxo de água em meios porosos deve-se resolver a Eq. 9 para todos os volumes de controle da malha computacional. Neste sentido, a literatura indica que o método mais utilizado é o método de aproximações sucessivas de Picard [8]. Contudo, como o problema que está sendo resolvido é não linear, a solução da Eq. 9 linearizada dá origem a um sistema linear de equações algébricas representadas pela Eq. 12:

$$
A\left(\psi^{m}\right) \psi^{m+1}=b\left(\psi^{m}\right)
$$

onde $A(\psi) \in \mathbb{R}^{\mathbb{N}} \times \mathbb{R}^{\mathbb{N}}$, enquanto $b(\psi)$ e $\psi \in \mathbb{R}^{\mathbb{N}}$; onde $\mathbb{N}$ é a dimensão dos vetores e $m$ o nível de iteração; também observa-se que $A(\psi)$ e $b(\psi)$ são funções da variável $\psi$ a ser determinada. 
Uma vez que normalmente o número de linhas da matriz $A(\psi)$ é igual ao número de nós do sistema de discretização espacial e, levando-se em conta que o número de nós pode ser da ordem de dezenas de milhares para uma aplicação em duas dimensões e da ordem de milhões para uma aplicação tridimensional e considerando-se que a matriz $A^{-1}(\psi)$ precisa ser determinada diversas vezes durante o processo de obtenção da solução, indubitavelmente solucionadores eficientes devem ser empregados. Em suma, visto que a resolução da Eq. 12 é a parte que consome o maior tempo de processamento, procedimentos iterativos devem ser adotados.

Por fim, pelos fatos evidenciados anteriormente e considerando que as técnicas iterativas mais modernas de resolução de sistemas de equações algébricas lineares são os métodos do subespaço de Krylov (KSP) combinados com matrizes pré-condicionadoras (PC) [3], o foco deste trabalho fundamentou-se em responder o seguinte questionamento:

Qual método de resolução de sistemas de equações algébricas lineares do subespaço de Krylov pré-condicionado que, acoplado às técnicas de Picard, é mais eficiente numérica e computacionalmente na solução da equação de Richards?

A resposta por sua vez não foi encontrada na literatura, apesar de existir algumas tentativas neste sentido.

\section{Validação do Código Computacional}

Para validar o código computacional foram resolvidos alguns problemas clássicos da literatura, a saber: Haverkamp et al. [11], Phoon et al. [7], Vasconcellos [2], Celia et al. [8] e Juncu et al. [5]. Igualmente foram utilizadas as soluções semi-analíticas de Philip [6], Warrick's et al. [1] e Tracy .

Em virtude dos resultados das simulações numéricas realizadas, conclui-se que o modelo numérico proposto modela bem problemas de curta infiltração e de longa duração. Por fim, considera-se que o programa computacional desenvolvido para resolver a equação de Richards baseada em $\psi$ em duas dimensões espaciais, a Eq. 2, está validado.

\section{Resultados e Discussões}

Os casos simulados propostos constituem-se basicamente de dois problemas de infiltração em perfis de solos homogêneos, no qual as especificações das propriedades hidráulicas foram obtidas em Bunsri, Sivakumar e Hagare [13].

\begin{tabular}{|c|c|c|c|c|c|c|}
\hline Solo & $K_{s}(\mathrm{~cm} / \mathrm{s})$ & $\theta_{r}$ & $\theta_{s}$ & $\alpha\left(\mathrm{cm}^{-1}\right)$ & $n$ & $\lambda$ \\
\hline Topsoil & $1,83889 \times 10^{-4}$ & 0,04 & 0,42 & 0,0249 & 1,6740 & 1,1045 \\
\hline Sand & $1,79611 \times 10^{-3}$ & 0,07 & 0,30 & 0,0446 & 2,1636 & 4,2424 \\
\hline
\end{tabular}

Tabela 1: Valores dos parâmetros das amostras de solo de Bunsri, Sivakumar e Hagare [13].

O primeiro problema a ser simulado, designado por IA, considera a infiltração de água numa coluna com dimensões $L_{z}=100 \mathrm{~cm}$ e $L_{x}=10 \mathrm{~cm}$, preenchida com o solo topsoil (vide Tab. 1), onde a condição inicial e as duas condições de contorno do tipo Dirichlet são fixadas pela Eq. 13:

$$
\begin{cases}\psi(z, 0)=-700,0 \mathrm{~cm}, & 0<z<100 \mathrm{~cm} \\ \psi(0, t)=-10,0 \mathrm{~cm}, & t>0 \\ \psi(100, t)=-700,0 \mathrm{~cm}, & t>0\end{cases}
$$

sendo que o fenômeno físico IA foi investigado durante um período de tempo de $6 h$. 
O segundo problema, nomeado de IB, considera o domínio de fluxo um quadrado com $L_{z}=100 \mathrm{~cm}$ e $L_{x}=100 \mathrm{~cm}$ e o solo $S a n d$, no qual os parâmetros hidráulicos estão definidos na Tab. 1. As condições iniciais e de contorno são estabelecidas pela Eq. 14:

$$
\begin{cases}t=0, & \theta(x, z, 0)=0,111 \\ z=0, & \theta(x, 0, t)=0,300 \\ z=L_{z}, & \theta\left(x, L_{z}, t\right)=0,111 \\ x=0, & \theta(0, z, t)=0,300 \\ x=L_{x}, & \theta\left(L_{x}, z, t\right)=0,111\end{cases}
$$

onde o fluxo de água foi medido para um período de tempo de 6 min. Como forma de ilustração a Fig. 2 retrata a evolução das frentes de molhamento do problema IB em diferentes tempos.

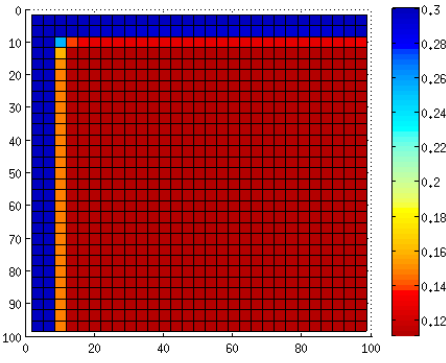

(a) $360 \mathrm{~s}$

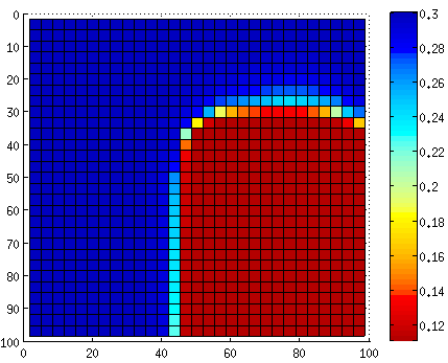

(c) $3600 \mathrm{~s}$

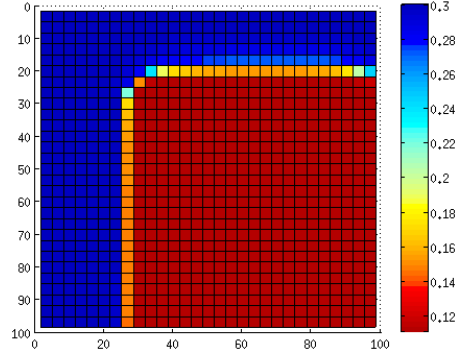

(e) $1800 \mathrm{~s}$

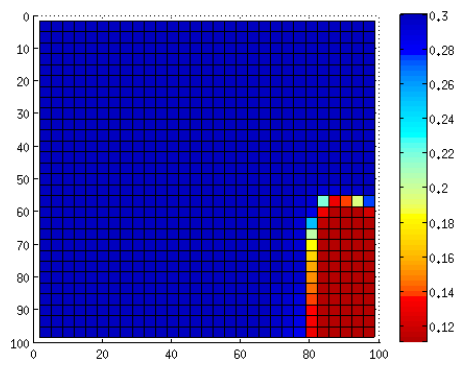

(g) $7200 \mathrm{~s}$

Figura 2: Evolução do teor de umidade volumétrico do experimento IB

Os resultados numéricos obtidos na simulação dos problemas IA e IB indicam que:

1. O tipo de solo e as condições físicas impostas podem influenciar o desempenho das combinações PICARD-KRYLOV-PC;

2. A solução do modelo de fluxo, independentemente dos modelos constitutivo acoplado, para ser resolvido em tempos computacionais aceitáveis, o sistema linear deve ser resolvido obrigatoriamente pela combinação KSPBCGS $\times$ PCSOR (Gradientes Biconjugados estabilizado mais o pré-condicionador SOR), uma vez que, para todos os testes realizados esta combinação esteve presente entre os melhores resultados;

3. Por outro lado, caso o modelo de fluxo seja resolvido apenas com as equações de van Genuchten, a escolha apropriada da combinação PICARD-KRYLOV-PC deve ser a KSPCG× PCSOR (Gradientes Conjugados mais o pré-condicionador SOR).

4. O fator determinante do tempo necessário para encontrar a solução da EDP de Richards, não é o número total de sistemas lineares resolvidos por passo de tempo, mas sim a velocidade de convergência do sistema linear a cada iteração. Neste sentido, o pré-condicionador SOR dentre os pré-condicionadores testados é o que melhor realiza a tarefa de aumentar a velocidade de convergência dos sistemas lineares; 
5. A combinação PICARD-KRYLOV-PC dada por KSPCGNE $\times$ PCBJACOBI (Gradientes Conjugados baseado nas Equações Normais mais o pré-condicionador bloco Jacobi) é a que apresentou os resultados menos satisfatórios, portanto inviabilizando a simulação de problemas de fluxo, independentemente dos modelos empíricos de Haverkamp et al. ou de van Genuchten;

\section{Referências}

[1] A. W. Warrick; D. O. Lomen; S. R. A. Yates, A generalized solution to infiltration, Soil Science Society of America Journal 49 (1985), no. 1, 34-38.

[2] C. A. B. de Vasconcellos; J. C. C Amorim, Numerical simulation of unsaturated flow in porous media using a mass-conservative model, Proceedings Brazilian Congress of Mechanical Engineering 8 (2001), 139-148.

[3] C. T. Miller, et al., Numerical simulation of water resources problems: Models, methods, and trends, Advances in Water Resources 51 (2013), no. 0, 405-437.

[4] F. T. Tracy, Three-dimensional analytical solutions of Richards' equation for a box-shaped soil sample with piecewise-constant head boundary conditions on the top, Journal of Hydrology 336 (2007), no. 3, 391-400.

[5] G. Juncu; A. Nicola; C. Popa, Nonlinear Multigrid Methods for Numerical Solution of the Variably Saturated Flow Equation in Two Space Dimensions, Transport in porous media 91 (2012), no. 1, 35-47.

[6] J. R. Philip, The Theory of Infiltration: 1. the Infiltration Equation and Its Solution, Soil Science 83 (1957), no. 5, 345-358.

[7] K. K. Phoon; et al., Numerical simulation of Richards equation in partially saturated porous media: under-relaxation and mass balance, Geotechnical and Geological Engineering 25 (2007), no. 5, 525-541.

[8] M. A. Celia; E. T. Bouloutas ; R. L. Zarba, A general mass-conservative numerical solution for the unsaturated flow equation, Water Resources Research 26 (1990), no. 7, 1483-1496.

[9] M. T. Van Genuchten, A Closed-form Equation for Predicting the Hydraulic Conductivity of Unsaturated Soil, Sci. Soc. Am. J. 44 (1980), 892-898.

[10] R. Haverkamp; M. Vauclin, A note on estimating finite difference interblock hydraulic conductivity values for transient unsaturated flow problems, Water Resources Research $\mathbf{1 5}$ (1979), no. 1, 181-187.

[11] R. Haverkamp; M. Vauclin; J. Touma; P. J. Wierenga; G. Vachaud, A Comparison of Numerical Simulation Models For One-Dimensional Infiltration1, 1977, pp. 285-294.

[12] S. Balay; J. Brown; K. Buschelman; W. D. Gropp; D. Kaushik; M. G. Knepley; L. C. Mcinnes; B. F. Smith; H. Zhang, PETSc Web page, 2013, http://www.mcs.anl.gov/petsc.

[13] T. Bunsri; M. Sivakumar; D. Hagare, Applications of Hydraulic Properties Models on Microscopic Flow in Unsaturated Porous Media, Journal of Applied Fluid Mechanics 2 (2009), no. $2,1-11$.

[14] Y. A. Mualem, A new model for predicting the hydraulic conductivity of unsaturated porous media, Water Resources Research 12 (1976), no. 3, 513-522. 